\title{
Autoimmunität in Pankreasinseln verläuft sehr unterschiedlich
}

Hintergrund und Fragestellung: Der Insulinmangel bei Typ1-Diabetes resultiert aus der Zerstörung der Langerhans'schen Inseln, die durch Immunzellen vermittelt wird. Daten zu Beschreibungen und Untersuchungen der Insulitis in den verschiedenen Stadien des (humanen) Prä-Typ-1-Diabetes, des manifesten Typ-1-Diabetes und des länger manifestierten Diabetes gibt es nur wenige, was der Grund für diese Studie war.

Patienten und Methoden: In dieser Studie wurde die Insulitisfrequenz (\% der Inseln mit Insulitis/gesamte Inseln), infiltrierende Leukozyten-Subpopulationen und die Betazell- und Al-

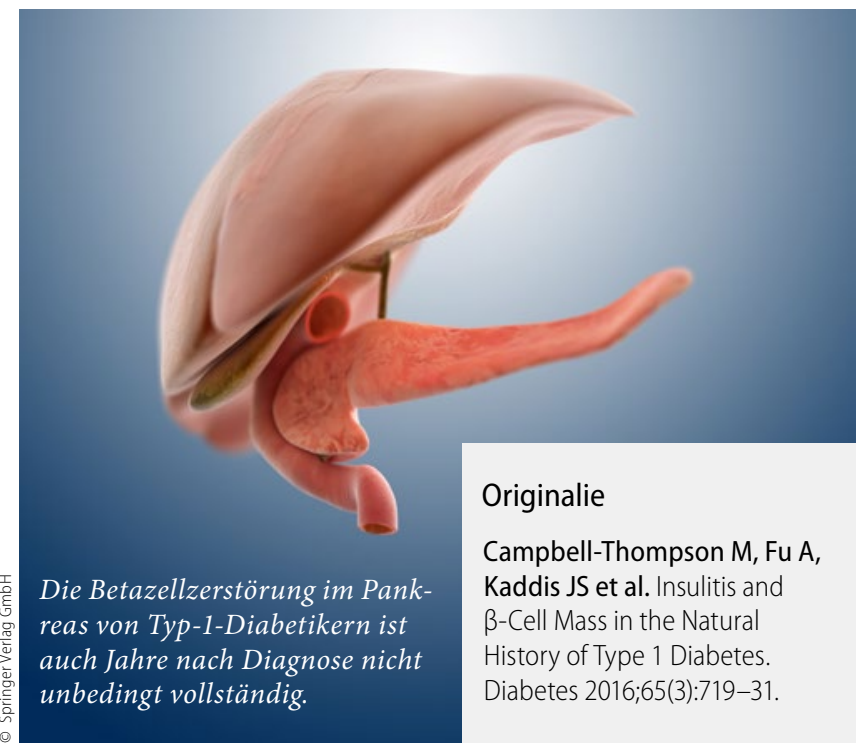

fazellmasse aus menschlichen Pankreata bestimmt. Die Organe stammten von Spendern, die Typ-1-Diabetes $(n=80)$ hatten oder keinen Diabetes hatten und dann entweder für Inselzellantikörper positiv $(\mathrm{AAb}(+), \mathrm{n}=18)$ oder negativ $(\mathrm{AAb}(-), \mathrm{n}=61)$ waren.

Ergebnisse: Eine Insulitis wurde bei 100\% (4/4) der Donoren gesehen, die eine Typ-1-Diabetes-Dauer von $\leq 1$ Jahr aufwiesen. Bei 11\% (2/18) der AAb(+) Organspender wurde ebenfalls eine Insulitis entdeckt, bei 89\% (16/18) der Aab (+) jedoch nicht. Die Insulitisfrequenz zeigte ein limitierte, aber inverse Korrelation mit der Diabetesdauer an $(r=-0,58, p=0,01)$, nicht aber mit dem Alter bei Diabetesdiagnose. Residuale Betazellen wurden bei allen Typ-1-Diabetes-Donoren gesehen, die eine Insulitis aufwiesen. Die Betazellfläche und Masse waren bei Typ-1-DiabetesDonoren mit Insulitis signifikant größer als bei Donoren ohne Insulitis. Die Insulitis betraf 33\% der insulinpositiven Inseln, und war lediglich bei $2 \%$ der insulinnegativen Inseln nachweisbar. Eine signifikante Korrelation wurde beobachtet für Insulitisfrequenz und CD45+, CD3+, CD4+, CD8+ und CD20+ Zellzahlen aus der Insulitis $(r=0,53-0,73, p=0,004-0,04)$. Eine signifikante Korrelation mit CD68+ oder CD11c+ wurde nicht gefunden.

Schlussfolgerungen: Die Präsenz sowohl von Betazellen als auch von Insulitis sogar noch mehrere Jahre nach Diabetesdiagnose bei Kindern und jungen Erwachsenen lässt darauf schließen, dass die Inselautoimmunität auch in der Zeit nach Diagnose noch voranschreitet. Diese Information sollte in Betracht gezogen werden, wenn man über therapeutische Strategien nachdenkt, mit denen ein Typ-1-Diabetes verhindert oder geheilt werden könnte.

\section{Ein neues Licht auf die pathogenetischen Veränderungen bei Insulitis}

Diese neuen Daten zeigen, dass die Insulitis beim natürlichen Verlauf des Typ-1-Diabetes nicht so fulminant wie im Tiermodell verläuft, längst nicht alle Inseln betrifft (auch nicht kurz

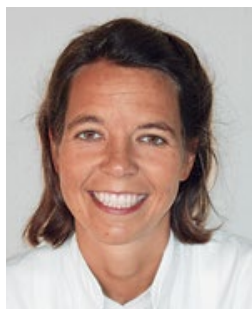

Prof. Dr. med. Nanette C. Schloot

Deutsches Diabetes-Zentrum,

Institut für Klinische Diabetologie an der Heinrich-Heine Universität Düsseldorf Nanette.Schloot@DDZ.uni-duesseldorf.de nach Diagnose), und auch noch viele Jahre nach Diagnose nachweisbar ist. Dass bedeutet auch, dass viele Jahre nach Manifestation noch intakte Inseln vorhanden sind. Während bei Organspendern, die nur einen positiven Inselzellautoantikörper aufwiesen, keine Insulitis nachweisbar war, zeigte sich bei $12 \%$ der Personen, die mehrfach antikörperpositiv waren, eine Insulitis.

Diese Ergebnisse sind überraschend und zeigen, dass vieles in der Pathogenese des Typ-1-Diabetes noch nicht verstanden ist. Mithilfe dieser neuen Daten aus der npod-Studie können zwar aufgrund des Settings keine longitudinalen Verläufe untersucht werden, aber allein die aktuellen Querschnittsuntersuchungen werfen ein neues Licht auf die pathogenetischen Veränderungen der Insulitis. 